\title{
The Monte-Carlo simulation on the detection efficiency of a scintillator neutron detector*
}

\author{
Fei Luo, Fang Liu ${ }^{\dagger}$, Ping Wang and Yu-Dong Sun \\ Beijing Key Laboratory of Passive Safety Technology for Nuclear Energy, \\ School of Nuclear Science and Engineering, \\ North China Electric Power University, \\ Beijing, 102206, China \\ 'E-mail: roffefly@163.com,liuf@ncepu.edu.cn
}

\begin{abstract}
We use Monte-Carlo simulation method to investigate several factors influencing detection efficiency of neutron detector. We have simulated the effect of crystal materials ( $\mathrm{ZnS}$ [1], $\mathrm{LiI}$ [2] and $\mathrm{CaF}_{2}$ [3]) on incident neutron flux and deposition energy. The result reveals that more energy deposited in the LiI (lithium iodide) scintillation crystal materials. And we explored the effect of the neutron source energy change on the detection efficiency of the scintillator. The simulation results show that the detection efficiency of the scintillation detector is gradually increased with the source energy increasing. These results will be beneficial to the development and application of neutron detectors
\end{abstract}

Keywords: Scintillation Detector; Detection Efficiency; Materials; Radioactive Source.

\section{Introduction}

The measurement of neutrons and their energy spectra is difficult because of the neutron itself without any charge. In order to detect neutrons, it is necessary to convert neutrons into charged particles by nuclear reactions, the method of which is used on the neutron detectors. Hydrogen-rich materials are often used as conversion materials, since ordinary hydrogen scatters neutrons which are converted into charged protons.

Detection efficiency is one of the important performance indexes of the detector. There are many factors that affect the efficiency of the detector. With full knowledge of these factors, we can adjust them to get the best detection efficiency. In this paper, we have discussed the detection efficiency from three aspects, the crystal materials, the source energy and the thickness of conversion medium. Results vary depending on the model used and on input data or

\footnotetext{
${ }^{*}$ This work was supported by the Project of National Natural Science Foundation of China (Grant No. 11405055) and the Fundamental Research Funds for the Central Universities (Grant No. 2014ZZD09).
} 
assumptions.
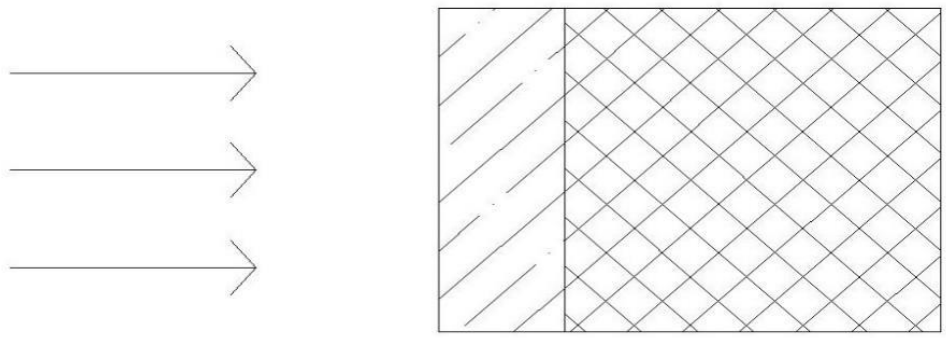

Fig.1. The diagram of crystal structure.

MCNP is a general-purpose, continuous-energy, generalized geometry, time-dependent, coupled neutron-photon-electron Monte Carlo transport code system [4]. For neutrons, code takes account of elastic scattering, inelastic scattering and capture reaction, according to the energy and direction of neutrons which have interaction with matters [5-6].

Table 1. The physical properties of simulation materials

\begin{tabular}{cccc}
\hline Substance & Density $(\mathrm{g} / \mathrm{cm} 3)$ & \multicolumn{2}{c}{ Atom Fraction } \\
\hline polyethylene & 0.93 & C: 0.333 & H: 0.667 \\
ZnS & 4.087 & Zn: 0.5 & S: 0.5 \\
LiI & 3.49 & Li: 0.5 & I: 0.5 \\
$\mathrm{CaF}_{2}$ & 3.18 & Ca: 0.333 & F: 0.667 \\
Air & 0.001205 & O:0.210750; N:0.784437 \\
& & C: $0.000151 ;$ Ar: 0.004671 \\
\hline
\end{tabular}

Diagram of the crystal structure simulation is showed in Figure 1. The geometric of crystal is cylinder without reflective layer. The crystal is divided into two parts, in which the neutron conversion layer is in front of the light-emitting layer. What's more, the materials of the light-emitting layer we choose include: $\mathrm{ZnS}$, LiI, $\mathrm{CaF}_{2}$, with the same material of neutron conversion layer (polyethylene). The physical properties of materials are showed in Table 1.The incident particles are generated by the event generator of the simulation program. And the neutron beam is emitted from the unidirectional parallel source, conversion in polyethylene and energy deposition in light-emitting layer. There is a certain relationship between the incident neutrons and the deposition energy in the scintillation crystal. By recording neutrons number through the incident interface and particles number depositing in the light-emitting layer, we could get the result of the detection efficiency of the scintillation crystal. 


\section{The Result and Discussion}

\subsection{The scintillation crystal materials}

Figure 2(a) gives the fluctuation of the number of incident neutrons particles in the case of different scintillation crystal materials. It is found that there are similar tendencies for the number of incident neutrons no matter what the materials of the crystal are. In addition, from Figure 2(b), we can find that under different materials of crystal, the number of deposition particles is not the same. When using LiI as the crystal material, the deposition particles are more than those others.
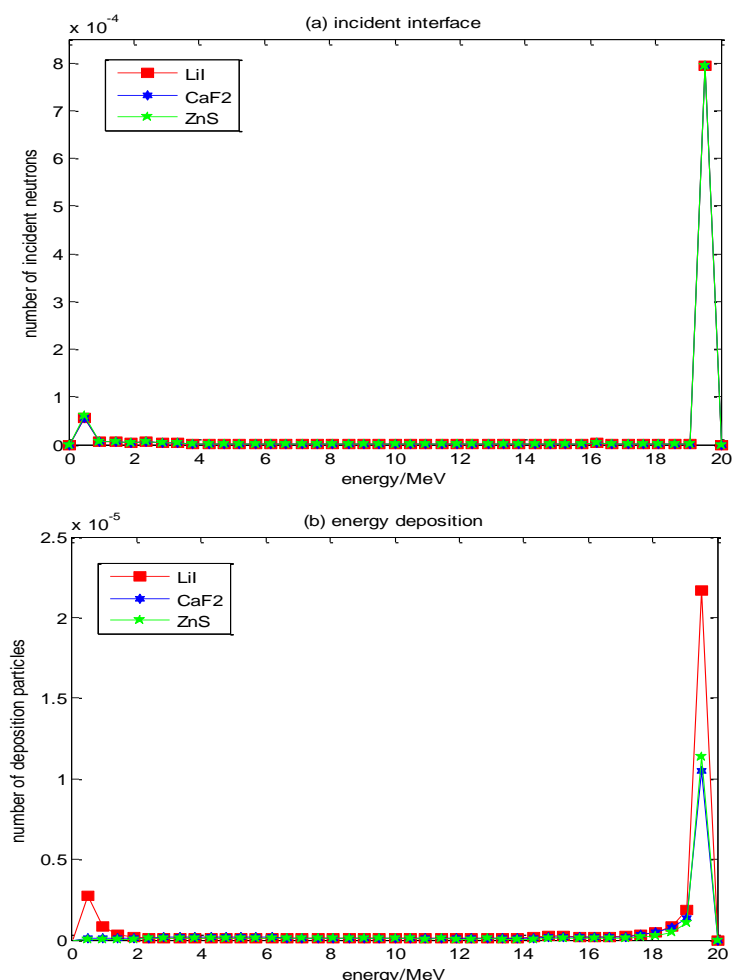

Fig. 2. The fluctuation of the number of incident neutrons and deposition particles in the case of different scintillation crystal materials

From the statistical results, it can be seen that the scintillation crystal materials have no effect on incident particles but on deposition particles. We think the reason leading to this situation is the interaction between the crystal materials and the particles converted from reaction of neutron with polyethylene. The degree of interaction between them is not identical. The reaction 
cross-sections of converted particles on crystal materials have determined the number of deposition particles. At the same time, we can read that the more particles deposit, the better detection efficiency is. For this, there is the better detection efficiency when using $\mathrm{LiI}$ as the crystal material than $\mathrm{ZnS}$ and $\mathrm{CaF}_{2}$.

\subsection{The source energy}

We performed many simulation experiments with a series of source energy. And the detection efficiency is calculated, and then the curves are plotted. From Figure 3, we can draw the following points. 1) The detection efficiency increases with the growth of the source energy. 2) When the source energy increases to a certain degree, the detection efficiency is expected to stabilize and climb no more. 3) The detection efficiency of lithium iodide is the best.

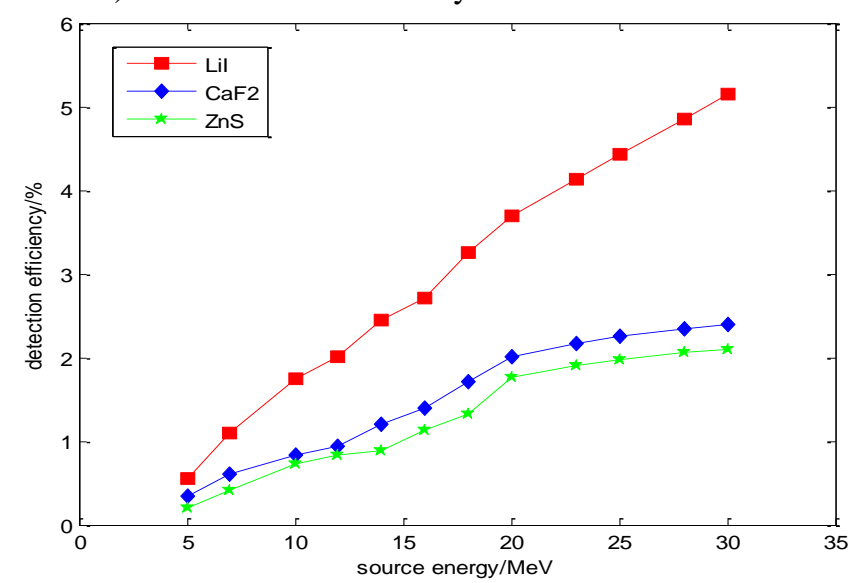

Fig. 3. The curve of detection efficiency with the change of source energy

We have got a discussion. The growth of incident neutrons energy increases the number of particles produced in the reaction between neutrons and the polyethylene conversion layer, which causes the raise of detection efficiency. There action cross-section, however, gradually reduces with the increase of neutrons energy. When the energy is enough, the neutrons will pass through the scintillation crystal without any reactions. Therefore, the detection efficiency is gradually stable.

\subsection{The thickness of polyethylene layer}

As Figure 4 shows, if polyethylene layer is too thin, the neutron may pass directly through the crystal without reaction. And if too thick, the converted particles may not be able to reach the light-emitting layer to deposit energy. In both cases, the detection efficiency is reduced due to the loss of particles. 
Thereby, we think the thickness of the polyethylene layer should be suitable to improve the detection efficiency.

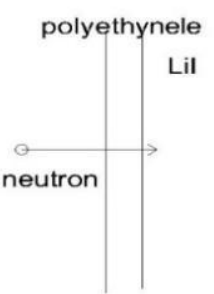

(1)

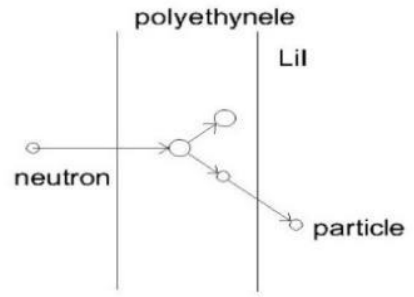

(2)

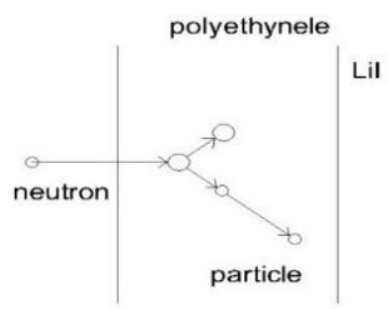

(3)

Fig. 4. The theoretical influence of the thickness of the polyethylene layer

We have simulated the influence of the polyethylene layer versus different thickness. Figure 5 gives the curve of detection efficiency with the change of the thickness of polyethylene layer. It is found that the efficiency of detector, with the increase of layer thickness, presents the first up and then down the fluctuation. What's more, there is the best detection efficiency on scintillators while the thickness of polyethylene layer is $1 \mathrm{~cm}$. Consequently, we can make sure that there exists a suitable thickness of the polyethylene layer, which makes the detection efficiency is the best.

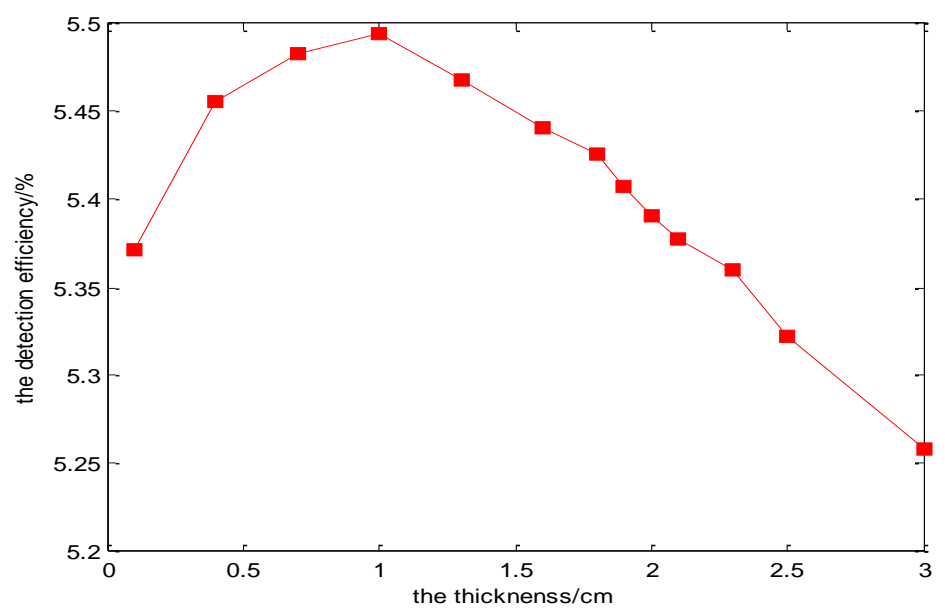

Fig. 5. The curve of detection efficiency with the change of the thickness of polyethylene layer

\section{Conclusion}

We make use of Monte Carlo simulation to investigate the influence of crystal materials, source energy and the conversion layer thickness on detection efficiency of neutron detector. It indicates that it can effectively improve the 
neutron detection efficiency when using a suitable material and lithium iodide can be a good choice. Furthermore, the source energy has a positive impact on the detection efficiency. Besides, for the polyethylene layer, there exists a suitable thickness which makes the detection efficiency is the best.

\section{References}

1. Tomotsu T, Hayakawa S, Makishima S. On ZnS Scintillator for Fast Neutron [J]. Oyobuturi, 1958, 27:38-41.

2. Syntfeld A, Moszyński M, Arlt R, et al. LiI(Eu) in Neutron and Gamma-ray Spectrometry a Highly Sensitive Thermal Neutron Detector[J]. IEEE Transactions on Nuclear Science, 2006, 52(6):3151-3156.

3. Rank E X, Theus R B. Neutron Dsosimetry with CaF2: Tm [J]. IEEE Transactions on Nuclear Science, 1979, 26(1):1590-1592.

4. Briesmeister J F. MCNP: A General Monte Carlo N-Particle Transport Code [J]. HepWebsearchHep, 1993

5. Varley F. Sears. Neutron scattering lengths and cross sections [J]. Methods of Experimental Physics, 1992, 3(3):26-37.

6. Bohr N. 3-Neutron Capture and Nuclear Constitution [J]. Nature, 1970, 137(3461):152-164. 